\title{
A Panel of Plasma Biomarkers for Differential Diagnosis of Parkinsonian Syndromes
}

\author{
Qi Lit, Zhen Lit, Xiaoxuan Han, Xiao Shen, Fei Wang, Lipeng Bai, Zhuo Li, Rui Zhang, \\ Yanlin Wang and Xiaodong Zhu*
}

Department of Neurology, Tianjin Neurological Institute, Tianjin Medical University General Hospital, Tianjin, China

Objective: The aim of our study is to explore the most reliable panel of plasma biomarkers for differential diagnosis of parkinsonian syndromes (PDSs). We selected five kinds of neurodegenerative proteins in plasma: neurofilament light chain (NfL), $\alpha$-synuclein ( $\alpha$-syn), total tau, $\beta$-amyloid 42 (A $\beta 42)$ and $\beta$-amyloid 40 (A $\beta 40)$, and investigated the diagnostic value of these biomarkers.

\section{OPEN ACCESS}

Edited by:

Pingyi Xu,

First Affiliated Hospital of Guangzhou Medical University, China

Reviewed by:

Shaogang Qu,

Southern Medical University, China

Yousheng Xiao,

Guangxi Medical University, China

*Correspondence:

Xiaodong Zhu

zxd3516@tmu.edu.cn

${ }^{t}$ These authors have contributed equally to this work

Specialty section:

This article was submitted to

Neurodegeneration,

a section of the journal

Frontiers in Neuroscience

Received: 31 October 2021

Accepted: 07 January 2022

Published: 17 February 2022

Citation:

Li Q, Li Z, Han X, Shen X, Wang F,

Bai L, Li Z, Zhang R, Wang Y and

Zhu X (2022) A Panel of Plasma Biomarkers for Differential Diagnosis of Parkinsonian Syndromes.

Front. Neurosci. 16:805953.

doi: 10.3389/fnins.2022.805953
Methods: A total of 99 plasma samples from patients with Parkinson's disease (PD), multiple system atrophy (MSA), progressive supranuclear palsy, and age-matched healthy controls (HCs) were enrolled in our study. Plasma NfL, $\alpha$-syn, total tau, A $\beta 42$, and $A \beta 40$ levels were quantified by ultrasensitive single molecule array immunoassay. We used logistic regression analyses to examine diagnostic accuracy of these plasma biomarkers. Disease severity was assessed by the modified Hoehn and Yahr staging scale, Unified Parkinson's Disease Rating Scale part III (UPDRS III), and the Mini-Mental State Examination (MMSE), and subsequently, correlation analysis was performed.

Results: A combination of $\alpha$-syn, $A \beta 42, A \beta 40, A \beta 42 / 40$, and $N f L$ could achieve a best diagnostic value in differentiating PDSs from $\mathrm{HC}$ and $\mathrm{PD}$ from $\mathrm{HC}$, with an AUC of 0.983 and 0.977 , respectively. By adding NfL to measurements of $\alpha$-syn or $A \beta 42$ or $A \beta 40$ or $A \beta 42 / 40$, the best discriminating panel was formed in differentiating atypical parkinsonian disorder (APD) and $\mathrm{HC}$, and the discriminatory potential could reach a sensitivity of $100 \%$ and specificity of $100 \%$ (AUC =1.000). For further distinguishing PD from APD, we found a combination of NfL, A $\beta 42$, and total tau was the most reliable panel with equally high diagnostic accuracy. With respect to differentiating the subtypes of APD from one another, our results revealed that measurement of NfL, total tau, $A \beta 42$, $A \beta 40$, and $A \beta 42 / 40$ was the best discriminating panel. Correlation analysis suggests that plasma A 42 levels were positively correlated to UPDRS part III scores in MSA. In terms of cognitive function, there was a relationship between plasma $A \beta 42 / 40$ level and MMSE scores in patients with APD.

Conclusion: In our study, various combinations of plasma biomarkers have great potentialities in identifying PDSs, with important clinical utility in improving diagnostic accuracy. Plasma NfL may have added value to a blood-based biomarker panel for differentiating PDSs.

Keywords: Parkinsonian syndromes, Parkinson's disease, biomarker, neurofilament light chain, panel 


\section{INTRODUCTION}

Parkinson's disease (PD) is one of the most common neurodegenerative disorders. Because of the large overlap of clinical symptoms, characterized by bradykinesia in combination with rest tremor, rigidity, or both (Geut et al., 2020), it is difficult to differentiate PD from atypical parkinsonian disorders (APDs) in early stages, which are mainly involved in multiple system atrophy (MSA) and progressive supranuclear palsy (PSP). There is an urgent need for reliable biomarkers to achieve an early and effective differential diagnosis in parkinsonian syndromes (PDSs).

Key factor that complicates the differentiation among different PDSs is the accompanying pathology. Generally, the neuropathologies of PDSs can be classified into two clusters, $\alpha$-synucleinopathy and tauopathy (Geut et al., 2020). It is universally acknowledged that a definite diagnosis with neuropathological confirmation is difficult to achieve. Therefore, biofluid biomarkers are the topical issues for the differential diagnoses. Several proteins associated with neurodegeneration, such as neurofilament light chain (NfL), $\alpha$-synuclein ( $\alpha$-syn), total tau, $\beta$-amyloid $42(\mathrm{~A} \beta 42)$ and $\beta$-amyloid 40 (A $\beta 40)$, have been wildly studied as potential biomarkers for PDSs in cerebrospinal fluid (CSF) (Marques et al., 2019; Parnetti et al., 2019; Oosterveld et al., 2020). It is valuable to investigate a convenient blood-based biomarker panel for differential diagnosis in PDSs.

The aim of this study was to investigate whether plasma NfL, $\alpha$-syn, total tau, $A \beta 42, A \beta 40$, and $A \beta 42 / A \beta 40$ contribute to an optimal biomarker panel to differentiate the subtypes of PDSs from one another and controls, quantified by an ultrasensitive single molecule array (Simoa) immunoassay. Furthermore, we performed the correlation analysis of plasma biomarkers with clinical features of disease severity in patients with PD and APD.

\section{MATERIALS AND METHODS}

\section{Study Participants}

All participants were recruited from the movement disorders outpatient clinics of the General Hospital of Tianjin Medical University. We analyzed a total of 99 plasma samples from patients with PD $(n=45)$, MSA $(n=13)$, PSP $(n=8)$, and age-matched healthy controls $(n=33)$. Inclusion criteria for PD met the United Kingdom Parkinson's Disease Society Brain Bank (UKPDSBB) criteria (Hughes et al., 1992). We defined patients entering the study as having MSA, following the "Second consensus statement on the diagnosis of MSA" (Gilman et al., 2008), and diagnosis of PSP patients was established according to the National Institute for Neurological Disorders and Stroke/Society criteria for PSP (Osaki et al., 2004). HCs who were recruited from the local community at the same period and matched for age and education have no history of serious physical, neurological, psychiatric disorders, and alcohol or substance abuse. The research protocols were approved and performed in accordance with the guidelines of the Ethics Committee of the General Hospital of Tianjin Medical
University. Written informed consent for scientific research purposes was obtained from all subjects.

\section{Clinical Evaluation}

The motor symptoms of patients were evaluated using the modified Hoehn and Yahr staging scale (H\&Y stage) (Hoehn and Yahr, 1967) and Unified Parkinson's Disease Rating Scale part III (UPDRS III) (Goetz et al., 2008) as measurements of clinical parkinsonian severity, which was performed during off medication, more than $12 \mathrm{~h}$ after the last dose of dopaminergic therapy. Global cognitive function was examined with the MiniMental State Examination (MMSE).

\section{Measurement of Plasma Biomarkers}

Fasting blood samples were collected by peripheral venous blood into $10-\mathrm{mL}$ EDTA tubes and centrifuged $(2,500 \mathrm{~g}$ for $15 \mathrm{~min}$ ) within $1 \mathrm{~h}$ after collection, aliquoted, and stored in polypropylene tubes at $-80^{\circ} \mathrm{C}$ until analysis. The plasma levels of NfL, $\alpha$-syn, total tau, $A \beta 42$, and $A \beta 40$ were measured by researchers who were blinded to the diagnosis and separately assayed via Simoa NfL Advantage kits (Quanterix, Lexington, MA, United States), Neurology 3Plex A advantage kit (lot 502473), and $\alpha$-syn discovery kit (Lot 502566) on the basis of manufacturer's introductions and standard procedures.

\section{Statistical Analyses}

All statistical analyses were performed with SPSS 22.0 (IBM, Inc., Armonk, NY, United States) and GraphPad Prism 9 (La Jolla, CA, United States). Shapiro-Wilk test was used to examine the Gaussian distribution of our data $(p>0.05)$. Numerical variables were displayed as mean $\pm \mathrm{SD}$. Comparisons of plasma biomarkers among different diagnostic groups were assessed by one-way analysis of variance (ANOVA), Kruskal-Wallis, and Mann-Whitney $U$ test. Multiple linear regression analyses adjusted for age, sex, and disease duration were conducted to examine the differences among diagnostic groups. The correlation among plasma proteins and clinical outcomes was accessed by Spearman rank correlation analysis. The diagnostic accuracy was examined with receiver operating characteristic (ROC) curve analyses. To determine the optimal differentiating biomarker panel, we performed a binary logistic regression analysis. $P<0.05$ was considered statistically significant.

\section{RESULTS}

\section{Clinical Characteristics}

A total of 99 participants consisting of 45 patients with PD, 13 patients with MSA, 8 patients with PSP, and 33 normal control subjects were enrolled in this study. Table 1 summarizes the characteristics and specific demographic data for all participants. The mean age of the four groups had no significant difference from each other $(p=0.05)$. In patients with APD, the motor severity (UPDRS part III scores and $\mathrm{H}-\mathrm{Y}$ stage) was significantly greater than that in $\mathrm{PD}(p<0.01)$. 
TABLE 1 | Clinical characteristics of study participants.

\begin{tabular}{|c|c|c|c|c|c|}
\hline Characteristics & $\mathrm{PD}(n=45)$ & MSA $(n=13)$ & $\operatorname{PSP}(n=8)$ & HC $(n=33)$ & $p$-value \\
\hline Male, \% & 48.89 & 78.57 & 62.50 & 51.52 & $<0.01^{\star *}$ \\
\hline Age, y & $66.15 \pm 4.75$ & $63.11 \pm 7.69$ & $70.00 \pm 7.31$ & $66.15 \pm 4.75$ & 0.05 \\
\hline Disease duration, y & $4.92 \pm 2.47$ & $2.22 \pm 0.67$ & $2.33 \pm 1.86$ & NA & $<0.01^{\star \star}$ \\
\hline Hoehn and Yahr stage & $1.31 \pm 0.53$ & $3.63 \pm 0.92$ & $3.17 \pm 0.41$ & NA & $<0.01^{\text {** }}$ \\
\hline MDS-UPDRS III (off) & $17.95 \pm 10.70$ & $42.54 \pm 18.00$ & $25.75 \pm 9.87$ & NA & $<0.01^{\star \star}$ \\
\hline MMSE & $28.43 \pm 1.86$ & $26.69 \pm 2.10$ & $25.00 \pm 5.34$ & NA & $<0.01^{\star \star}$ \\
\hline $\mathrm{NfL}(\mathrm{pg} / \mathrm{mL})$ & $20.43 \pm 14.09$ & $86.53 \pm 33.74$ & $53.90 \pm 22.97$ & $16.00 \pm 5.18$ & $<0.01^{\star \star}$ \\
\hline Total tau (pg/mL) & $1.08 \pm 0.66$ & $0.51 \pm 0.18$ & $0.55 \pm 0.17$ & $0.93 \pm 0.63$ & $<0.01^{\star \star}$ \\
\hline $\mathrm{A} \beta 42(\mathrm{pg} / \mathrm{mL})$ & $10.10 \pm 2.99$ & $5.30 \pm 1.17$ & $5.83 \pm 2.28$ & $5.26 \pm 2.01$ & $<0.01^{* *}$ \\
\hline $\mathrm{A} \beta 40(\mathrm{pg} / \mathrm{mL})$ & $167.35 \pm 49.41$ & $119.50 \pm 23.78$ & $136.39 \pm 30.76$ & $103.90 \pm 31.00$ & $<0.01^{\star \star}$ \\
\hline$A \beta 42 / 40$ & $0.04 \pm 0.01$ & $0.04 \pm 0.01$ & $0.04 \pm 0.01$ & $0.05 \pm 0.01$ & $<0.01^{\star *}$ \\
\hline$\alpha$-synuclein (pg/mL) & $43.50 \pm 33.63$ & $44.01 \pm 18.51$ & $53.75 \pm 52.18$ & $10.36 \pm 5.51$ & $<0.01^{\star *}$ \\
\hline
\end{tabular}

Data are presented as mean $\pm S D$. The $p$-values were obtained from comparisons among four groups of participants using analysis of variance.

${ }^{* *} p<0.01$.

PD, Parkinson's disease; MSA, multiple system atrophy; PSP, progressive supranuclear palsy; HC, healthy controls; MMSE, Mini-Mental State Examination; NfL,

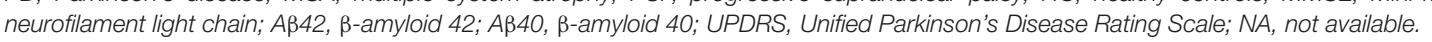

\section{Plasma Biomarker Levels in Different Diagnostic Groups}

Mean plasma biomarker levels among different diagnostic groups are presented in Table $\mathbf{1}$. We compared individual plasma biomarker levels of normal controls and in different diagnostic groups. To adjust for the potential confounding factors of age, sex, and disease duration, we performed multiple linear regression. After adjustment, we found that the plasma $A \beta 40$, $\mathrm{A} \beta 42 / 40$, and $\alpha$-syn levels were significantly higher in the PD group compared with HCs (A $\beta 40:$ PD vs. HC: $p<0.0001$; A $\beta 42 / 40:$ PD vs. HC: $p<0.001 ; \alpha$-syn: PD vs. HC: $p<0.0001$; Figures 1C-E). The levels of plasma total tau, $A \beta 42$, and $A \beta 42 / 40$ were significantly elevated in patients with $\mathrm{PD}$ compared with those in APD (total tau: PD vs. MSA: $p<0.01$, PD vs. PSP: $p<0.05$; A $\beta 42$ : PD vs. MSA: $p<0.0001$, PD vs. PSP: $p<0.001$; A $\beta 42 / 40$ : PD vs. MSA: $p<0.0001$, PD vs. PSP: $p<0.0001$; Figures 1A,B,D). Moreover, the level of plasma NfL was significantly increased in patients with MSA and PSP when compared with PD (PSP, $p<0.0001$; MSA, $p<0.0001$ ) and controls (PSP, $p<0.0001$; MSA, $p<0.0001$ ). Plasma NfL concentrations in patients with MSA were clearly higher than those in PSP ( $p=0.02$ ) (Figure 1F). Only patients with PSP had an increased expression level of $A \beta 40$ and $\alpha$-syn compared with controls (A $\beta 40: p<0.05$; $\alpha$-syn: $p<0.0001$, Figures 1C,E).

\section{Diagnostic Accuracy of Plasma Biomarkers}

Discriminatory value of plasma NfL, total tau, $\mathrm{A} \beta 42, \mathrm{~A} \beta 40$, $\mathrm{A} \beta 42 / 40$ and $\alpha$-syn as a single biomarker and as part of plasma biomarker panel in differentiating PDSs (PD and APD) from $\mathrm{HC}$ is presented in Table 2. The biomarkers were selected to combine as a panel according to the level of AUC; that is, we selected the biomarkers to combine as a panel according to the ability of independently predicted diagnosis of individual biomarker.

\section{Parkinsonian Syndromes vs. Healthy Controls}

Plasma $\alpha$-syn showed $80 \%$ sensitivity and $93.9 \%$ specificity with an AUC of 0.889 for differentiating PDSs (PD, MSA, and PSP) from $\mathrm{HC}$, with the highest diagnostic value among individual biomarkers, whereas a combination of $\mathrm{A} \beta 42, \mathrm{~A} \beta 40, \mathrm{~A} \beta 42 / 40$, $\mathrm{NfL}$, and $\alpha$-syn could achieve a better diagnostic value in differentiating PDSs from HC with an AUC of 0.983 with $98.5 \%$ sensitivity and $93.9 \%$ specificity. However, the panel of $\alpha$-syn, $\mathrm{A} \beta 40$, and NfL could achieve a relatively desired effect with the minimum types of markers (an AUC of 0.980 with a sensitivity and specificity of 93.8 and $97.0 \%$ ). In order to differentiate $\mathrm{PD}$ subjects from $\mathrm{HC}$, we found $\mathrm{A} \beta 42$ as the dominant single biomarker revealed an AUC of 0.925 , with a sensitivity and specificity of 93.1 and $87.9 \%$. There was very good discriminatory power between PD groups and HC with an AUC of 0.977 with a sensitivity and specificity of 97.8 and $93.9 \%$ using the panel of $\mathrm{A} \beta 42, \mathrm{~A} \beta 40, \mathrm{~A} \beta 42 / 40, \mathrm{NfL}$, and $\alpha$-syn, the same as the panel for discriminating PDSs and HC. The combination of $\mathrm{A} \beta 42, \mathrm{~A} \beta 40$, and $\alpha$-syn was also a good choice to discriminating PD from $\mathrm{HC}$ with the highest specificity up to $97 \%$ (AUC = 0.972). When looking into the APD (MSA and PSP) groups, NfL exhibited very good discriminatory power in differentiating MSA and PSP from $\mathrm{HC}$ with an AUC of 1.000 and 0.996, respectively. By adding NfL to measurement of $\alpha$-syn or $A \beta 42$ or $A \beta 40$ or $A \beta 42 / 40$, the best discriminating panel was formed in differentiating APD and $\mathrm{HC}$ with a sensitivity of $100 \%$ and specificity of $100 \%$ $($ AUC $=1.000$; Table 2$)$, implying NfL was the extraordinarily desirable biomarker for differentiating APD from HC.

\section{Parkinson's Disease vs. Atypical Parkinsonian Disorder}

The ROC curve analysis revealed that NfL was the most reliable biomarker to differentiate PD from APD, which had a sensitivity of $88.9 \%$ and a specificity of $95.0 \%$ with an AUC of 0.963 . When we looked into differentiating PD from MSA and PSP, NfL still exhibited very good discriminatory power with an AUC of 0.983 and 0.993 , respectively (PD vs. MSA: a sensitivity and 
A

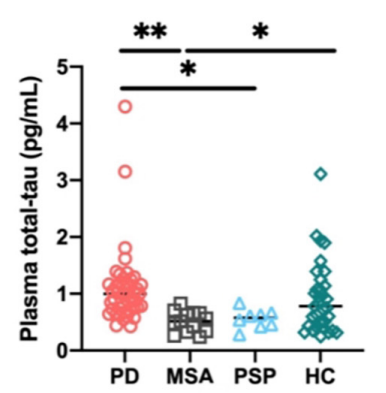

D

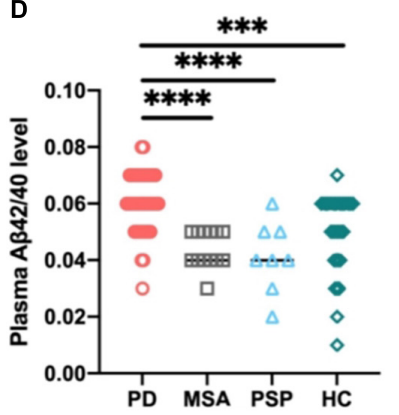

B

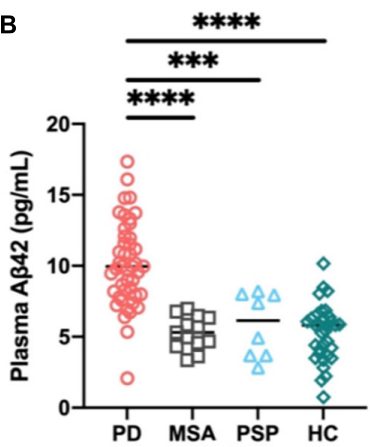

E

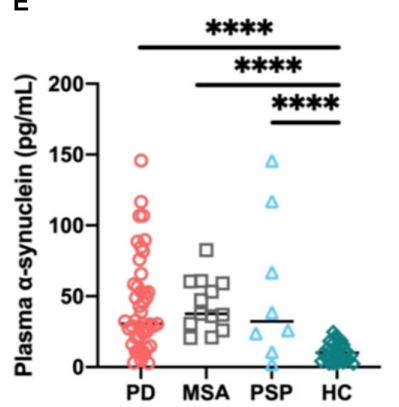

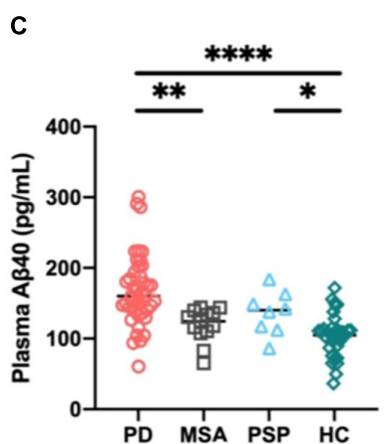

$\mathbf{F}$

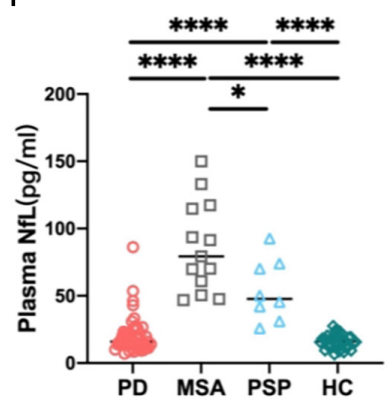

FIGURE 1 | Individual plasma biomarker levels of normal controls and in different diagnostic groups. The plasma total tau (A), A 342 (B), and Aß42/40 (D) levels were

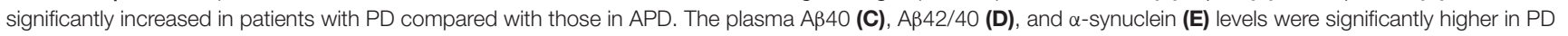
group compared with healthy controls. The plasma NfL (F) level was significantly increased in patients with MSA and PSP compared with PD and controls. $p$-values are from multiple linear regression models adjusting for age, sex, and disease duration. PD, Parkinson's disease; MSA, multiple system atrophy; PSP, progressive supranuclear palsy; $\mathrm{HC}$, healthy controls; NfL, neurofilament light chain; $A \beta 42, \beta$-amyloid $42 ; A \beta 40, \beta$-amyloid 40; $A \beta 42 / 40, \beta$-amyloid $42 / 40$; **** $p<0.0001$,

${ }^{* * *} p<0.001,{ }^{* *} p<0.01,{ }^{*} p<0.05$.

specificity of 100 and $95.6 \%$, respectively; PD vs. PSP: a sensitivity and specificity of 82.5 and $100 \%$, respectively). As seen from Table 3, the best differentiating panel between PD and APD was formed by the combination of plasma NfL, A $\beta 42$, and total tau, which yielded an ideal sensitivity and specificity of $100 \%$ (AUC $=1.000)$, whereas the combination of $\mathrm{A} \beta 42$ and total tau without NfL revealed an AUC of 0.963 with a sensitivity of $95 \%$ and a specificity of $91.1 \%$.

\section{Multiple System Atrophy vs. Progressive Supranuclear Palsy}

Neurofilament light was the most reliable biomarker to differentiate MSA from PSP, which had a sensitivity of $78.3 \%$ and a specificity of $87.5 \%(\mathrm{AUC}=0.802$; Table 4$)$. The model of total tau, $A \beta 42, A \beta 40$, and $A \beta 42 / 40$ could differentiate MSA from PSP with an AUC of 0.823 . Now adding plasma NfL into the model, the best discriminatory panel was formed by the combination of NfL, total tau, $A \beta 42, A \beta 40$, and $A \beta 42 / 40$ with an AUC of 1.000, a sensitivity of $100 \%$, and a specificity of $100 \%$.

A summary of the best single biomarker and panel in comparing different PDSs from one another and controls is presented in Table 5.

\section{Correlation Between Plasma Biomarkers and Motor Severity}

We investigated whether there was a relationship between plasma biomarkers and motor severity as measured by MDS-UPDRS part III and H\&Y stage. According to Table 1, patients with APD had significantly worse motor dysfunction compared with PD patients ( $p<0.01$ by ANOVA). Correlation analysis revealed that plasma A $\beta 42$ levels were positively correlated to UPDRS part III scores in patients with MSA ( $r=0.589, p=0.03$; Figure 2$)$. Other biomarkers were not correlated with UPDRS part III during "off” time in PDS patients.

\section{Correlation Between Plasma Biomarkers and Cognitive Function}

Previous studies have confirmed that $\beta$-amyloid contributed to the prediction of cognitive deterioration in other neurodegenerative diseases, just as $\mathrm{PD}$, not limited to Alzheimer disease $(\mathrm{AD})$. We next investigated the relevance between plasma biomarkers and cognitive status (MMSE) in each diagnostic group. By means of correlation analysis, we found that plasma A $342 / 40$ level positively correlated with MMSE scores only in patients with APD (MSA: $r=0.583, p=0.03$; PSP: $r=0.763$, $p=0.03$; Figure 3).

\section{DISCUSSION}

In this study, we aimed to demonstrate whether plasma levels of NfL, $\alpha$-syn, total tau, $A \beta 42, A \beta 40$, and $A \beta 42 / 40$ integrated could be applied to identify PDSs. For this purpose, we measured the levels of several specific and meaningful biomarkers in 
TABLE 2 | Discriminatory value of plasma NfL, total tau, $A \beta 42, A \beta 40, A \beta 42 / 40$ and $\alpha$-syn as a single biomarker and as part of plasma biomarker panel in differentiating PDS (PD and APD) from $\mathrm{HC}$.

\begin{tabular}{|c|c|c|c|c|}
\hline $\begin{array}{l}\text { Diagnostic } \\
\text { groups }\end{array}$ & Predictor & AUC & Sensitivity & Specificity \\
\hline \multirow[t]{12}{*}{ PDS vs. HC } & Single biomarker & & & \\
\hline & $\alpha-S y n$ & 0.889 & $80.0 \%$ & $93.9 \%$ \\
\hline & $A \beta 40$ & 0.824 & $83.1 \%$ & $78.8 \%$ \\
\hline & $A \beta 42$ & 0.800 & $70.8 \%$ & $87.9 \%$ \\
\hline & NfL & 0.694 & $46.2 \%$ & $97.0 \%$ \\
\hline & $A \beta 42 / 40$ & 0.576 & $48.5 \%$ & $51.5 \%$ \\
\hline & Total tau & 0.513 & $83.1 \%$ & $33.3 \%$ \\
\hline & Biomarker panel & & & \\
\hline & $\begin{array}{l}\text { A } \beta 42, A \beta 40, A \beta 42 / 40, N f L, \text { and } \\
\alpha-s y n\end{array}$ & 0.983 & $98.5 \%$ & $93.9 \%$ \\
\hline & $\alpha-S y n, A \beta 40, A \beta 42 / 40$, and NfL & 0.982 & $96.9 \%$ & $93.9 \%$ \\
\hline & $\alpha$-Syn, A $\beta 40$, and NfL & 0.980 & $93.8 \%$ & $97.0 \%$ \\
\hline & $\alpha-S y n$, total tau, and NfL & 0.921 & $73.8 \%$ & $100 \%$ \\
\hline \multirow[t]{11}{*}{ PD vs. HC } & Single biomarker & & & \\
\hline & $\mathrm{A} \beta 42$ & 0.925 & $93.1 \%$ & $87.9 \%$ \\
\hline & $\alpha-S y n$ & 0.875 & $73.3 \%$ & $97.0 \%$ \\
\hline & $\mathrm{A} \beta 40$ & 0.873 & $88.9 \%$ & $78.8 \%$ \\
\hline & $\mathrm{A} \beta 42 / 40$ & 0.736 & $53.3 \%$ & $90.9 \%$ \\
\hline & Total tau & 0.612 & $42.4 \%$ & $86.7 \%$ \\
\hline & NfL & 0.558 & $33.3 \%$ & $87.9 \%$ \\
\hline & Biomarker panel & & & \\
\hline & $\begin{array}{l}\mathrm{A} \beta 42, \mathrm{~A} \beta 40, \mathrm{~A} \beta 42 / 40, \mathrm{NfL}, \text { and } \\
\alpha \text {-syn }\end{array}$ & 0.977 & $97.8 \%$ & $93.9 \%$ \\
\hline & $\begin{array}{l}\text { A } \beta 42, A \beta 40, A \beta 42 / 40, \text { and } \\
\alpha \text {-syn }\end{array}$ & 0.974 & $95.6 \%$ & $97.0 \%$ \\
\hline & $\mathrm{A} \beta 42, \mathrm{~A} \beta 40$, and $\alpha-\operatorname{syn}$ & 0.972 & $95.6 \%$ & $97.0 \%$ \\
\hline \multirow[t]{13}{*}{ APD vs. HC } & Single biomarker & & & \\
\hline & NfL & 0.998 & $100 \%$ & $97.0 \%$ \\
\hline & $\alpha-S y n$ & 0.920 & $90.3 \%$ & $93.9 \%$ \\
\hline & $A \beta 42 / 40$ & 0.721 & $80 \%$ & $81.8 \%$ \\
\hline & $A \beta 40$ & 0.715 & $70.6 \%$ & $78.8 \%$ \\
\hline & Total tau & 0.711 & $75.0 \%$ & $75.8 \%$ \\
\hline & $\mathrm{A} \beta 42$ & 0.521 & $35.0 \%$ & $76.8 \%$ \\
\hline & Biomarker panel & & & \\
\hline & NfL and $\alpha$-syn & 1.000 & $100 \%$ & $100 \%$ \\
\hline & NfL and $A \beta 40$ & 1.000 & $100 \%$ & $100 \%$ \\
\hline & NfL and $A \beta 42$ & 1.000 & $100 \%$ & $100 \%$ \\
\hline & NfL and $A \beta 42 / 40$ & 1.000 & $100 \%$ & $100 \%$ \\
\hline & NfL and total tau & 0.998 & $100 \%$ & $97.0 \%$ \\
\hline \multirow[t]{7}{*}{ MSA vs. HC } & Single biomarker & & & \\
\hline & $\mathrm{NfL}$ & 1.000 & $100 \%$ & $100 \%$ \\
\hline & $\alpha-S y n$ & 0.990 & $100 \%$ & $93.9 \%$ \\
\hline & Total tau & 0.737 & $66.7 \%$ & $75.8 \%$ \\
\hline & $\mathrm{A} \beta 42 / 40$ & 0.720 & $66.7 \%$ & $75.8 \%$ \\
\hline & $A \beta 40$ & 0.667 & $83.3 \%$ & $54.5 \%$ \\
\hline & $\mathrm{A} \beta 42$ & 0.513 & $58.3 \%$ & $57.6 \%$ \\
\hline \multirow[t]{5}{*}{ PSP vs. HC } & Single biomarker & & & \\
\hline & NfL & 0.996 & $100 \%$ & $100 \%$ \\
\hline & $\alpha-S y n$ & 0.814 & $100 \%$ & $93.9 \%$ \\
\hline & $\mathrm{A} \beta 40$ & 0.788 & $83.3 \%$ & $54.5 \%$ \\
\hline & $\mathrm{A} \beta 42 / 40$ & 0.723 & $66.7 \%$ & $75.8 \%$ \\
\hline
\end{tabular}

TABLE 2 | (Continued)

\begin{tabular}{llccc}
\hline $\begin{array}{l}\text { Diagnostic } \\
\text { groups }\end{array}$ & Predictor & AUC & Sensitivity & Specificity \\
\hline & & & & \\
\hline & Total tau & 0.670 & $66.7 \%$ & $75.8 \%$ \\
& A $\beta 42$ & 0.572 & $58.3 \%$ & $42.4 \%$ \\
& Biomarker panel & & \\
NfL and $\alpha$-syn & 1.000 & $100 \%$ & $100 \%$ \\
& NfL and A $\beta 40$ & 1.000 & $100 \%$ & $100 \%$ \\
& NfL and A $\beta 42$ & 1.000 & $100 \%$ & $100 \%$ \\
& NfL and A $\beta 42 / 40$ & 1.000 & $100 \%$ & $100 \%$ \\
& NfL and total tau & 0.996 & $100 \%$ & $97.0 \%$
\end{tabular}

PDS, parkinsonian syndrome; APD, atypical parkinsonian disorders; $P D$, Parkinson's disease; MSA, multiple system atrophy; PSP, progressive supranuclear palsy; HC, healthy controls; NfL, neurofilament light chain; A $\beta 42, \beta$-amyloid 42; A $\beta 40, \beta$-amyloid 40; A $\beta 42 / 40, \beta$-amyloid 42/40; AUC, area under the curve; $\alpha$-syn, $\alpha$-synuclein.

plasma quantified by high-sensitivity immunoassays. Our results indicated that plasma NfL was significantly increased, and plasma total tau, $A \beta 42$, and $A \beta 42 / 40$ levels were lower in patients with APD than those in PD. NfL is an extraordinarily prospective biomarker as a single measure for differentiating APD (MSA and PSP) from PD and HCs as well as differentiating MSA from PSP, with high sensitivity and specificity. By means of adding NfL to the biomarker panel, the diagnostic accuracy of dramatically improved on visual inspection. According to our results, various combinations of these six biomarkers were able to differentiate PDSs from HC, APD from PD, and the subtypes of APD from one another. Furthermore, we performed the correlation analysis suggesting that there was a correlation observed between plasma $A \beta$ with motor and cognitive function in APD. Together, a measurement of integrated biomarker panel has great potentialities of differential diagnosis in PDSs, and these potential biomarkers may be indicators of monitoring motor and cognitive progression in patients with PDSs.

$\mathrm{NfL}$ is the most important and abundant subunit in neurofilaments. Under normal conditions, NfL is released from axons, probably in an age-dependent manner, and increases with age (Disanto et al., 2017). However, on special occasions in response to CNS axonal damage, such as inflammatory, neurodegenerative, traumatic, or vascular injury, the release of NfL is notably rising (Gaetani et al., 2019). Our findings are consistent with those reported in previous studies (Gaetani et al., 2019; Marques et al., 2019; Archer et al., 2020). All the relevant studies mentioned previously verified that plasma NfL was significantly higher in APD than that in PD and thus proved to have diagnostic value in identifying APD.

Definitely, plasma and CSF amyloid $\beta$ (ratio of amyloid $\beta 42$ to amyloid $\beta 40$ ) reflect brain amyloidosis (Nakamura et al., 2018; Schindler et al., 2019). A $\beta$ peptides are important for neuronal information processing and are major constituents of amyloid plaques deposited in the brains of patients with Alzheimer's disease (AD), but also seen in other neurodegenerative diseases and notably in patients accompanied with dementia (Gomperts et al., 2013). In this study, we 
TABLE 3 | Discriminatory value of plasma NfL, total tau, $A \beta 42, A \beta 40, A \beta 42 / 40$ and $\alpha$-syn as a single biomarker and as part of plasma biomarker panel in differentiating PD from APD (MSA and PSP).

\begin{tabular}{|c|c|c|c|c|}
\hline $\begin{array}{l}\text { Diagnostic } \\
\text { groups }\end{array}$ & Predictor & AUC & Sensitivity & Specificity \\
\hline \multirow[t]{12}{*}{ PD vs. APD } & Single biomarker & & & \\
\hline & $\mathrm{NfL}$ & 0.963 & $88.9 \%$ & $95.0 \%$ \\
\hline & $A \beta 42$ & 0.924 & $71.1 \%$ & $100 \%$ \\
\hline & Total tau & 0.914 & $80.0 \%$ & $95.0 \%$ \\
\hline & $\mathrm{A} \beta 42 / 40$ & 0.897 & $73.0 \%$ & $100 \%$ \\
\hline & $\mathrm{A} \beta 40$ & 0.791 & $73.3 \%$ & $85.0 \%$ \\
\hline & $\alpha-\operatorname{Syn}$ & 0.562 & $55.6 \%$ & $65.6 \%$ \\
\hline & Biomarker panel & & & \\
\hline & $\mathrm{NfL}, \mathrm{A} \beta 42$ and total tau & 1.000 & $100 \%$ & $100 \%$ \\
\hline & NfL and total tau & 0.996 & $95.6 \%$ & $100 \%$ \\
\hline & $\mathrm{NfL}, \mathrm{A} \beta 42$ and $A \beta 42 / 40$ & 0.990 & $100 \%$ & $95.6 \%$ \\
\hline & $A \beta 42$, total tau, and $A \beta 42 / 40$ & 0.981 & $100 \%$ & $88.9 \%$ \\
\hline \multirow[t]{11}{*}{ PD vs. MSA } & Single biomarker & & & \\
\hline & $\mathrm{NfL}$ & 0.983 & $100 \%$ & $95.6 \%$ \\
\hline & $\mathrm{A} \beta 42$ & 0.961 & $88.9 \%$ & $100 \%$ \\
\hline & Total tau & 0.930 & $80.0 \%$ & $100 \%$ \\
\hline & $\mathrm{A} \beta 42 / 40$ & 0.896 & $77.8 \%$ & $100 \%$ \\
\hline & $\mathrm{A} \beta 40$ & 0.850 & $78.9 \%$ & $100 \%$ \\
\hline & $\alpha-\operatorname{Syn}$ & 0.600 & $55.6 \%$ & $65.0 \%$ \\
\hline & Biomarker panel & & & \\
\hline & NfL, total tau, and $A \beta 42 / 40$ & 1.000 & $100 \%$ & $100 \%$ \\
\hline & NfL and total tau & 0.998 & $100 \%$ & $97.8 \%$ \\
\hline & A $\beta 42$ and total tau & 0.985 & $91.1 \%$ & $100 \%$ \\
\hline \multirow[t]{13}{*}{ PD vs. PSP } & Single biomarker & & & \\
\hline & $\mathrm{NfL}$ & 0.993 & $82.5 \%$ & $100 \%$ \\
\hline & $\mathrm{A} \beta 42 / 40$ & 0.897 & $73.3 \%$ & $100 \%$ \\
\hline & Total tau & 0.892 & $82.2 \%$ & $87.5 \%$ \\
\hline & $\mathrm{A} \beta 42$ & 0.869 & $71.1 \%$ & $100 \%$ \\
\hline & $A \beta 40$ & 0.703 & $62.2 \%$ & $75.0 \%$ \\
\hline & $\alpha-\operatorname{Syn}$ & 0.506 & $62.3 \%$ & $37.5 \%$ \\
\hline & Biomarker panel & & & \\
\hline & $\mathrm{NfL}, \mathrm{A} \beta 42 / 40$, and total tau & 1.000 & $100 \%$ & $100 \%$ \\
\hline & NfL, total tau, and $A \beta 42$ & 1.000 & $100 \%$ & $100 \%$ \\
\hline & $\mathrm{NfL}$ and total tau & 0.992 & $95.6 \%$ & $100 \%$ \\
\hline & $\mathrm{NfL}$ and $\mathrm{A} \beta 42 / 40$ & 0.978 & $95.6 \%$ & $100 \%$ \\
\hline & $\mathrm{NfL}$ and $\mathrm{A} \beta 42$ & 0.967 & $93.3 \%$ & $100 \%$ \\
\hline
\end{tabular}

APD, atypical parkinsonian disorders; PD, Parkinson's disease; MSA, multiple system atrophy; PSP, progressive supranuclear palsy; HC, healthy controls; NfL, neurofilament light chain; $A \beta 42, \beta$-amyloid 42; $A \beta 40, \beta$-amyloid 40; $A \beta 42 / 40$, $\beta$-amyloid 42/40; AUC, area under the curve; $\alpha$-syn, $\alpha$-synuclein.

found plasma amyloid $\beta$ was significantly higher in PDS compared with controls, consistent with those reported in previous studies (Seino et al., 2019). Moreover, our observations that plasma A $\beta 42 / 40$ level positively correlated with global cognitive status in patients with APD further confirmed the close connection between amyloid $\beta$ and cognitive dysfunction. However, another finding revealed that plasma A $\beta 42$ levels were positively correlated to UPDRS part III in patients with MSA, despite no enough evidence for the correlation at present.
TABLE 4 | Discriminatory value of plasma NfL, total tau, A $\beta 42, A \beta 40, A \beta 42 / 40$ and $\alpha$-syn as a single biomarker and as part of plasma biomarker panel in differentiating MSA from PSP.

\begin{tabular}{|c|c|c|c|c|}
\hline $\begin{array}{l}\text { Diagnostic } \\
\text { groups }\end{array}$ & Predictor & AUC & Sensitivity & Specificity \\
\hline \multirow[t]{12}{*}{ MSA vs. PSP } & Single biomarker & & & \\
\hline & $\mathrm{NfL}$ & 0.802 & $78.3 \%$ & $87.5 \%$ \\
\hline & $\mathrm{A} \beta 40$ & 0.698 & $83.3 \%$ & $62.5 \%$ \\
\hline & $\mathrm{A} \beta 42$ & 0.594 & $100 \%$ & $50.0 \%$ \\
\hline & Total tau & 0.583 & $82.2 \%$ & $57.5 \%$ \\
\hline & $\mathrm{A} \beta 42 / 40$ & 0.573 & $58.5 \%$ & $63.8 \%$ \\
\hline & $\alpha-S y n$ & 0.551 & $50.0 \%$ & $67.6 \%$ \\
\hline & Biomarker panel & & & \\
\hline & $\begin{array}{l}\mathrm{NfL}, \text { total tau, } A \beta 42, A \beta 40 \text {, } \\
\text { and } A \beta 42 / 40\end{array}$ & 1.000 & $100 \%$ & $100 \%$ \\
\hline & $\begin{array}{l}\text { NfL, total tau, } A \beta 42, A \beta 40 \text {, } \\
\text { A } \beta 42 / 40 \text {, and } \alpha \text {-syn }\end{array}$ & 1.000 & $100 \%$ & $100 \%$ \\
\hline & $\begin{array}{l}\text { NfL, total tau, } A \beta 42 \text {, and } \\
\text { A } \beta 40\end{array}$ & 0.844 & $83.3 \%$ & $87.5 \%$ \\
\hline & $\begin{array}{l}\text { Total tau, } A \beta 42, A \beta 40 \text {, and } \\
A \beta 42 / 40\end{array}$ & 0.823 & $91.7 \%$ & $62.5 \%$ \\
\hline
\end{tabular}

MSA, multiple system atrophy; PSP, progressive supranuclear palsy; $H C$, healthy

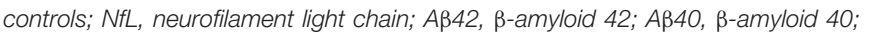
A $42 / 40, \beta$-amyloid 42/40; AUC, area under the curve; $\alpha$-syn, $\alpha$-synuclein.

TABLE 5 | The best single biomarker and panel in comparing different parkinsonian syndromes with each other and controls.

\begin{tabular}{|c|c|c|c|c|}
\hline $\begin{array}{l}\text { Diagnostic } \\
\text { groups }\end{array}$ & $\begin{array}{c}\text { Single } \\
\text { biomarker }\end{array}$ & AUC & Panel & AUC \\
\hline PDS-HC & $\alpha-S y n$ & 0.889 & $\begin{array}{l}\text { A } \beta 42, A \beta 40, A \beta 42 / 40, N f L, \text { and } \\
\alpha \text {-syn }\end{array}$ & 0.983 \\
\hline PD-HC & $\mathrm{A} \beta 42$ & 0.925 & $\begin{array}{l}\text { A } \beta 42, A \beta 40, A \beta 42 / 40, N f L, \text { and } \\
\alpha-s y n\end{array}$ & 0.977 \\
\hline APD-HC & $\mathrm{NfL}$ & 0.998 & $\begin{array}{l}\text { NfL and } \alpha \text {-syn or } A \beta 42 \text { or } A \beta 40 \\
\text { or } A \beta 42 / 40\end{array}$ & 1 \\
\hline MSA-HC & $\mathrm{NfL}$ & 1 & NA & NA \\
\hline PSP-HC & $\mathrm{NfL}$ & 0.996 & $\begin{array}{l}\text { NfL and } \alpha \text {-syn or } A \beta 42 \text { or } A \beta 40 \\
\text { or } A \beta 4240\end{array}$ & 1 \\
\hline PD-APD & $\mathrm{NfL}$ & 0.963 & $\mathrm{NfL}, \mathrm{A} \beta 42$ and total tau & 1 \\
\hline PD-MSA & $\mathrm{NfL}$ & 0.983 & $\mathrm{NfL}, \mathrm{A} \beta 42 / 40$, and total tau & 1 \\
\hline PD-PSP & $\mathrm{NfL}$ & 0.993 & $\begin{array}{l}\text { NfL, total tau, and } A \beta 42 / 40 \text { or } \\
A \beta 42\end{array}$ & 1 \\
\hline MSA-PSP & $\mathrm{NfL}$ & 0.802 & $\begin{array}{l}\text { NfL, total tau, } A \beta 42, A \beta 40 \text {, and } \\
\text { A } \beta 42 / 40\end{array}$ & 1 \\
\hline
\end{tabular}

PDS, parkinsonian syndrome; $A P D$, atypical parkinsonian disorders; $P D$, Parkinson's disease; MSA, multiple system atrophy; PSP, progressive supranuclear palsy; HC, healthy controls; NfL, neurofilament light chain; $A \beta 42, \beta$-amyloid 42; A $\beta 40, \beta$-amyloid 40; A $\beta 42 / 40$, $\beta$-amyloid 42/40; AUC, area under the curve; $\alpha$-syn, $\alpha$-synuclein.; NA, not available.

The major component of neurofibrillary tangles is the natively unfolded phosphoprotein tau. In human neurodegenerative diseases with filamentous deposits, such as $\mathrm{AD}$, tau protein becomes hyperphosphorylated, as an initiating event preceding filament assembly (Lee et al., 2001). The imbalance of the tau isoforms with three (3R-tau) or four (4R-tau) microtubule-binding repeat domains contributes to the pathogenesis of neurodegeneration. Particularly, PSP is a $4 \mathrm{R}$ 


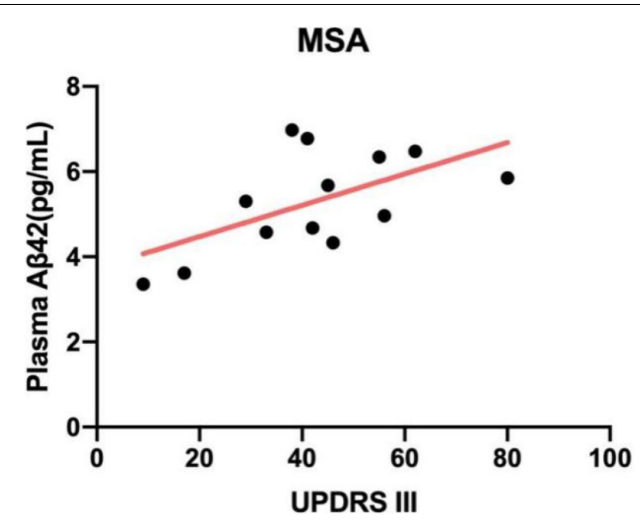

FIGURE 2 | Correlation between plasma A 342 and UPDRS part III scores in patients with MSA. The levels of plasma $A \beta 42$ were positively related to UPDRS part III ( $r=0.589, p=0.03)$. MSA, multiple system atrophy; $A \beta 42$, $\beta$-amyloid 42; UPDRS part III, Unified Parkinson's Disease Rating Scale part III.

tau neuropathological entity. Several studies have reported that elevated total tau levels in CSF may help to distinguish PDSs (Süssmuth et al., 2010; Aerts et al., 2011), whereas no such comparable findings were observed in blood (Lin et al., 2018; Li et al., 2021). Furthermore, total tau may be an indicator of cognition. Total tau protein levels have been applied to discriminate dementia with Lewy bodies and PD dementia (PDD) from AD (Howard et al., 2021). However, in our study, measurement of plasma total tau alone was not able to achieve good diagnostic value. Possible explanations may be due to the limited number of patients enrolled and deficient measurement of phosphorylated tau protein.

Our results highlight that plasma NfL is an extraordinarily prospective biomarker as a single measure for differentiating PDSs, whereas other plasma biomarker measurements could not reach an ideal diagnostic value. However, a panel of plasma biomarkers was proven to make great improvement in diagnostic accuracy. A combination of $\alpha$-syn, $A \beta 42, A \beta 40, A \beta 42 / 40$, and NfL could achieve a best diagnostic value in differentiating
PDS from HC and PD from HC. As we speculated, by adding NfL to measurements of other biomarker models, there was an obvious improvement in differentiating APD patients from PD and HC. When adding NfL to measurements of $\alpha$-syn, $A \beta 42$, $\mathrm{A} \beta 40$, or $\mathrm{A} \beta 42 / 40$, the best discriminating panel was formed in differentiating APD and HC with a sensitivity of $100 \%$ and specificity of $100 \%$, and combination of NfL, A $\beta 42$, and total tau was proved to be the most reliable panel for differential diagnosis between $\mathrm{PD}$ and $\mathrm{APD}$, with equally high diagnostic accuracy. For differentiating the subtypes of APD from one another, our results revealed that measurement of NfL, total tau, $\mathrm{A} \beta 42, \mathrm{~A} \beta 40$, and $\mathrm{A} \beta 42 / 40$ was the best discriminating panel. Our findings of improved discriminatory potential using a biomarker panel confirm that the combination of NfL with other plasma biomarkers could differentiate PDSs more accurately than a single blood biomarker.

The major strength of our present study was that we evaluated the diagnostic value of several specific plasma biomarkers (NfL, total tau, $\mathrm{A} \beta 42, \mathrm{~A} \beta 40, \mathrm{~A} \beta 42 / 40$ and $\alpha$-syn) for discriminating PDSs. Combining multiple blood-based biomarkers considerably increases the diagnostic sensitivity and specificity. Simultaneously, we conducted the correlation analysis of plasma biomarkers with motor symptom and cognition. However, there are some limitations to our present study. First, final diagnoses of our participants were established based on clinical evaluations rather than neuropathological confirmation, which increased the possibility of misclassification. Second, the relatively restricted number of patients and lack of follow-up and tracking the changes of biomarkers over time may limit the clinical application to PDS patients. Third, there were significant differences in clinical characteristics among different groups, for which we interpret that the diagnostic groups we enrolled are heterogeneous; namely, the disease severity is diverse among different PDSs, embodied in motor symptoms (H\&Y stage, MDSUPDRS III) and non-motor features (cognitive impairment, sleep disorders, and mood disturbances). For this reason, bias may arise. Finally, a combination of other candidate biomarkers, including CSF, serum, saliva, and skin, would be an enhancement in diagnosis accuracy.
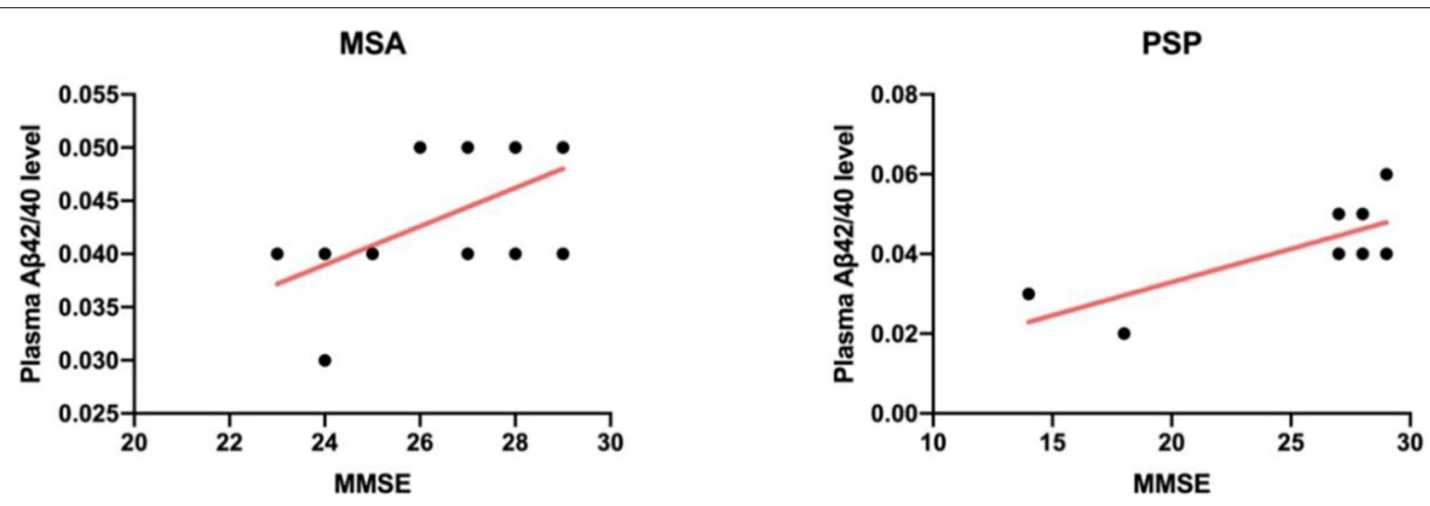

FIGURE 3 | Scatter plot showing the relationship between plasma A $42 / 40$ level and MMSE scores in the MSA group (Left) and PSP group (Right). Solid line indicates a correlation observed between AB42/40 level and global cognitive status in patients with APD (MSA: $r=0.583, p=0.03 ; \mathrm{PSP}: r=0.763, p=0.03$ ). MSA, multiple system atrophy; PSP, progressive supranuclear palsy; Aß42/40, $\beta$-amyloid 42/40; MMSE, Mini-Mental State Examination. 


\section{CONCLUSION}

Our findings support the use of a panel of biomarkers to differentiate PDSs from HC, APD from PD, and the subtypes of APD from one another. Plasma NfL is an extraordinarily prospective biomarker contributing to a biomarker panel in discriminating APD patients from PD and $\mathrm{HC}$ and discriminating different subtypes of APD. This outcome may have essential clinical benefit in terms of improving diagnostic accuracy of PDSs. Further large-scale, longitudinal follow-up studies are needed to evaluate the differential value of these integrated plasma biomarkers and validate whether plasma biomarkers levels could be applied to predict progression of motor and non-motor symptoms in patients with PDSs.

\section{DATA AVAILABILITY STATEMENT}

The original contributions presented in the study are included in the article/supplementary material, further inquiries can be directed to the corresponding author.

\section{ETHICS STATEMENT}

The studies involving human participants were reviewed and approved by the Ethics Committee of the General Hospital of Tianjin Medical University. The

\section{REFERENCES}

Aerts, M. B., Esselink, R. A., Bloem, B. R., and Verbeek, M. M. (2011). Cerebrospinal fluid tau and phosphorylated tau protein are elevated in corticobasal syndrome. Mov. Disord. 26, 169-173. doi: 10.1002/mds. 23341

Archer, D. B., Mitchell, T., Burciu, R. G., Yang, J., Nigro, S., Quattrone, A., et al. (2020). Magnetic Resonance Imaging and Neurofilament Light in the Differentiation of Parkinsonism. Mov. Disord. 35, 1388-1395. doi: 10.1002/mds. 28060

Disanto, G., Barro, C., Benkert, P., Naegelin, Y., Schädelin, S., Giardiello, A., et al. (2017). Swiss Multiple Sclerosis Cohort Study Group. Serum Neurofilament light: a biomarker of neuronal damage in multiple sclerosis. Ann. Neurol. 81, 857-870. doi: 10.1002/ana.24954

Gaetani, L., Blennow, K., Calabresi, P., Di Filippo, M., Parnetti, L., and Zetterberg, H. (2019). Neurofilament light chain as a biomarker in neurological disorders. J. Neurol. Neurosurg. Psychiatry 90, 870-881. doi: 10.1136/jnnp-2018-320106

Geut, H., Hepp, D. H., Foncke, E., Berendse, H. W., Rozemuller, J. M., Huitinga, I., et al. (2020). Neuropathological correlates of parkinsonian disorders in a large Dutch autopsy series. Acta Neuropathol. Commun. 8:39. doi: 10.1186/s40478020-00914-9

Gilman, S., Wenning, G. K., Low, P. A., Brooks, D. J., Mathias, C. J., Trojanowski, J. Q., et al. (2008). Second consensus statement on the diagnosis of multiple system atrophy. Neurology 71, 670-676. doi: 10.1212/01.wnl.0000324625.00404. 15

Goetz, C. G., Tilley, B. C., Shaftman, S. R., Stebbins, G. T., Fahn, S., MartinezMartin, P., et al. (2008). Movement Disorder Society UPDRS Revision Task Force. Movement Disorder Society-sponsored revision of the Unified Parkinson's Disease Rating Scale (MDS-UPDRS): scale presentation and clinimetric testing results. Mov. Disord. 23, 2129-2170. doi: 10.1002/mds.22340 patients/participants provided their written informed consent to participate in this study.

\section{AUTHOR CONTRIBUTIONS}

QL: conceptualization, methodology, software, formal analysis, writing - original draft, and writing - review and editing. ZheL: investigation, resources, methodology, data curation, software, and writing-original draft. XH, XS, FW, LB, ZhuL, $\mathrm{RZ}$, and YW: investigation, validation, and writing - review and editing. XZ: supervision, project administration, and funding acquisition. All authors read and approved the final version of the manuscript.

\section{FUNDING}

This work was supported by Science \& Technology Development Fund of Tianjin Education Commission for Higher Education under grants 2017ZD10, Tianjin Natural Science Foundation under grants 19JCZDJC35300, and Tianjin Health Commission under grants KJ20009.

\section{ACKNOWLEDGMENTS}

QL and ZheL are the first author in parallel. The collection and sharing of data for this study were done by ZheL, and the processing and analysis of data were completed by QL.

Gomperts, S. N., Locascio, J. J., Rentz, D., Santarlasci, A., Marquie, M., Johnson, K. A., et al. (2013). Amyloid is linked to cognitive decline in patients with Parkinson's disease without dementia. Neurology 80, 85-91. doi: 10.1212/WNL. 0b013e31827b1a07

Hoehn, M. M., and Yahr, M. D. (1967). Parkinsonism: onset, progression and mortality. Neurology 17, 427-442. doi: 10.1212/wnl.17.5.427

Howard, E., Irwin, D. J., Rascovsky, K., Nevler, N., Shellikeri, S., Tropea, T. F., et al. (2021). Cognitive Profile and Markers of Alzheimer Disease-Type Pathology in Patients With Lewy Body Dementias. Neurology 96, e1855-e1864. doi: 10.1212/ WNL.0000000000011699

Hughes, A. J., Daniel, S. E., Kilford, L., and Lees, A. J. (1992). Accuracy of clinical diagnosis of idiopathic Parkinson's disease: a clinico-pathological study of 100 cases. J. Neurol. Neurosurg. Psychiatry 55, 181-184. doi: 10.1136/jnnp.55. 3.181

Lee, V. M., Goedert, M., and Trojanowski, J. Q. (2001). Neurodegenerative tauopathies. Annu. Rev. Neurosci. 24, 1121-1159. doi: 10.1146/annurev.neuro. 24.1.1121

Li, C. H., Chen, T. F., Chiu, M. J., Yen, R. F., Shih, M. C., and Lin, C. H. (2021). Integrated 18F-T807 Tau PET, Structural MRI, and Plasma Tau in Tauopathy Neurodegenerative Disorders. Front. Aging Neurosci. 13:646440. doi: 10.3389/ fnagi.2021.646440

Lin, C. H., Yang, S. Y., Horng, H. E., Yang, C. C., Chieh, J. J., Chen, H. H., et al. (2018). Plasma Biomarkers Differentiate Parkinson's Disease From Atypical Parkinsonism Syndromes. Front. Aging Neurosci. 10:123. doi: 10.3389/fnagi. 2018.00123

Marques, T. M., van Rumund, A., Oeckl, P., Kuiperij, H. B., Esselink, R. A. J., Bloem, B. R., et al. (2019). Serum NFL discriminates Parkinson's disease from atypical parkinsonisms. Neurology 92, e1479-e1486. doi: 10.1212/WNL. 0000000000007179 
Nakamura, A., Kaneko, N., Villemagne, V. L., Kato, T., Doecke, J., Doré, V., et al. (2018). High performance plasma amyloid- $\beta$ biomarkers for Alzheimer's disease. Nature 554, 249-254. doi: 10.1038/nature 25456

Oosterveld, L. P., Verberk, I. M. W., Majbour, N. K., El-Agnaf, O. M., Weinstein, H. C., Berendse, H. W., et al. (2020). CSF or serum neurofilament light added to $\alpha$-Synuclein panel discriminates Parkinson's from controls. Mov. Disord. 35, 288-295. doi: 10.1002/mds.27897

Osaki, Y., Ben-Shlomo, Y., Lees, A. J., Daniel, S. E., Colosimo, C., Wenning, G., et al. (2004). Accuracy of clinical diagnosis of progressive supranuclear palsy. Mov. Disord. 19, 181-189. doi: 10.1002/mds.10680

Parnetti, L., Gaetani, L., Eusebi, P., Paciotti, S., Hansson, O., El-Agnaf, O., et al. (2019). CSF and blood biomarkers for Parkinson's disease. Lancet Neurol. 18, 573-586. doi: 10.1016/S1474-4422(19)30024-9

Schindler, S. E., Bollinger, J. G., Ovod, V., Mawuenyega, K. G., Li, Y., Gordon, B. A., et al. (2019). High-precision plasma $\beta$-amyloid $42 / 40$ predicts current and future brain amyloidosis. Neurology 93, e1647-e1659. doi: 10.1212/WNL. 0000000000008081

Seino, Y., Nakamura, T., Kawarabayashi, T., Hirohata, M., Narita, S., Wakasaya, Y., et al. (2019). Cerebrospinal Fluid and Plasma Biomarkers in Neurodegenerative Diseases. J. Alzheimers Dis. 68, 395-404. doi: 10.3233/JAD-18 1152
Süssmuth, S. D., Uttner, I., Landwehrmeyer, B., Pinkhardt, E. H., Brettschneider, J., Petzold, A., et al. (2010). Differential pattern of brain-specific CSF proteins tau and amyloid- $\beta$ in Parkinsonian syndromes. Mov. Disord. 25, 1284-1288. doi: $10.1002 / \mathrm{mds} .22895$

Conflict of Interest: The authors declare that the research was conducted in the absence of any commercial or financial relationships that could be construed as a potential conflict of interest.

Publisher's Note: All claims expressed in this article are solely those of the authors and do not necessarily represent those of their affiliated organizations, or those of the publisher, the editors and the reviewers. Any product that may be evaluated in this article, or claim that may be made by its manufacturer, is not guaranteed or endorsed by the publisher.

Copyright (C) 2022 Li, Li, Han, Shen, Wang, Bai, Li, Zhang, Wang and Zhu. This is an open-access article distributed under the terms of the Creative Commons Attribution License (CC BY). The use, distribution or reproduction in other forums is permitted, provided the original author(s) and the copyright owner(s) are credited and that the original publication in this journal is cited, in accordance with accepted academic practice. No use, distribution or reproduction is permitted which does not comply with these terms. 